\title{
Actividades lúdicas para el mejoramiento de la lectura comprensiva en estudiantes de educación básica
}

\author{
Playful Activities for the Improvement of Reading Comprehension in Elementary School \\ Students
}

\section{Actividades lúdicas para a melhoria da compreensão da leitura em alunos do ensino básico}

ARTÍCULO DE INVESTIGACIÓN

\author{
Gina Venegas Álvarez ${ }^{1}$ \\ gina.venegas@utc.edu.ec \\ https://orcid.org/0000-0001-8356-6162 \\ Sonia Castro Bungacho ${ }^{1}$ \\ sonia.castro@utc.edu.ec \\ https://orcid.org/0000-0003-4500-0541 \\ Carlos Proaño Rodríguez ${ }^{2}$ \\ ceproanio@espe.edu.ec \\ https://orcid.org/0000-0002-9709-4634 \\ Gina Tello Cóndor ${ }^{3}$ \\ ginatello1979@hotmail.com \\ https://orcid.org/0000-0002-9709-4634

\section{Universidad Técnica de Cotopaxi, Cotopaxi Ecuador \\ ${ }^{2}$ Universidad de las Fuerzas Armadas ESPE, Cotopaxi Ecuador ${ }^{3}$ Unidad Educativa Básica Numa Pompilio Llona, Cotopaxi Ecuador}

Recibido 08 de enero 2021 | Arbitrado y aceptado 15 de febrero 2021 | Publicado en 01 abril 2021

\section{RESUMEN}

El objetivo que persigue este estudio es identificar las principales necesidades presentes en un grupo de estudiantes de Secundaria Básica en cuanto al nivel de lectura comprensiva que poseen y partiendo de esto proponer un programa de actividades lúdicas para lograr el mejoramiento de esta habilidad, tomando en consideración que las actividades lúdicas representan una herramienta pedagógica que contribuye a potenciar la adquisición de conocimientos y el desarrollo de habilidades en el educando, mejorando aspectos como la motivación, la predisposición, la participación, activa y además permite relacionar los contenidos abordados con cargas emocionales positivas. La metodología empleada en el desarrollo de la investigación se enmarca en el enfoque cualitativo-cuantitativo, por medio de la aplicación de la técnica de la encuesta dirigida a los docentes y a los estudiantes, complementado con la modalidad aplicada puesto que se desarrolló una propuesta ajustada a las dimensiones y a las características de la problemática dentro de la Escuela de Educación Básica Numa Pompilio Llona. Los principales resultados, evidencian que el $50 \%$ de estudiantes leen poco, el $70 \%$ prefieren leer cuentos, el $90 \%$ prefieren que su docente fomente la lectura a través de actividades lúdicas y el $40 \%$ de ellos refiere falta de comprensión en la lectura.

Palabras clave: Lectura; Comprensión; Juego; Aprendizaje

\section{ABSTRACT}

The objective of this study is to identify the main needs present in a group of elementary school students in terms of their comprehension reading level and, based on this, to propose a series of recreational activities to achieve the improvement of this skill, taking into consideration that recreational activities represent a pedagogical tool that contributes to enhance the acquisition of knowledge and the development of skills in the student, improving aspects such as motivation, predisposition, active participation, and also allows relating the contents addressed with positive emotional charges. The methodology used in the development of the research is framed in the qualitative-quantitative approach, through the application of the survey technique directed to teachers and students, complemented with the applied modality since a proposal adjusted to the dimensions and characteristics of the problem within the School of Basic Education Numa Pompilio Llona was developed. The main results show that $50 \%$ of the students read little, $70 \%$ prefer to read stories, $90 \%$ prefer their teacher to encourage reading through playful activities and $40 \%$ of them refer to a lack of reading comprehension.

Key words: Reading; Comprehension; Play; Learning

\section{RESUMO}

0 objectivo deste estudo é identificar as principais necessidades presentes num grupo de alunos do Ensino Secundário Básico em termos do nível de leitura abrangente que possuem e, com base nisso, propor uma série de actividades lúdicas para alcançar a melhoria desta competência, tendo em consideração que as actividades lúdicas representam uma ferramenta pedagógica que ajuda a melhorar a aquisição de conhecimentos e o desenvolvimento de competências no aluno, melhorando aspectos como a motivação, a predisposição, a participação, a actividade e também permite relacionar os conteúdos abordados com cargas emocionais positivas. A metodologia utilizada no desenvolvimento da investigação está enquadrada na abordagem qualitativa-quantitativa, através da aplicação da técnica do inquérito dirigido aos professores e aos alunos, complementada com a modalidade aplicada, uma vez que foi desenvolvida uma proposta ajustada às dimensões e às características da problemática dentro da Escola do Ensino Básico Numa Pompilio Llona. Os principais resultados mostram que $50 \%$ dos alunos lêem pouco, $70 \%$ preferem ler histórias, $90 \%$ preferem que o seu professor incentive a leitura através de actividades lúdicas e $40 \%$ deles referem a falta de compreensão de leitura.

Palavras-Chave: Leitura; Compreensão; Ludopatia; aprendizagem 


\section{INTRODUCCIÓN}

Desde la antigüedad, los juegos se han convertido en parte de la vida cotidiana de las diferentes especies que viven en la tierra, como se ha comprobado a través de diversas investigaciones, el juego con animales y el juego humano, son los diferentes aspectos de esta actividad en la vida social, darle a conocer otros conceptos y métodos para colocarlos en práctica, y aplicar reglas o definir estrategias de participación en muchas ocasiones. Así, la importancia de crear espacios donde las actividades de ocio sean parte del proceso de enseñanza y aprendizaje, que es otra forma de impartir conocimientos, crear experiencia previa o reforzar la experiencia ya adquirida.

La expresión lúdica es una actividad antigua, no solo correspondiente a los humanos (Huizinga, 1968), señaló: "Los juegos son más antiguos que la cultura; bueno, por muy estrecho que sea su concepto, siempre presupone la sociedad humana".

Sin embargo, las actividades lúdicas han ganado espacio en el proceso educativo, (Zuluaga \& Gómez, 2016) insistió: "Enseñar a través del juego es una propuesta para contribuir a potenciar las habilidades de los estudiantes en el aula". Por ello, Domínguez, (2015) señaló que es fundamental en la educación actual cuente en el desarrollo psicológico del juego de los niños.

A medida que avanza el mundo, los juegos han cambiado de los populares juegos tradicionales al uso de nuevas tecnologías como los juegos virtuales. Por ejemplo, los juegos que se encuentran en las aplicaciones móviles de hoy se pueden distinguir entre sí. Algunas personas que ayudan a lograr los objetivos de entretenimiento están a favor de lograr una mejor educación, mientras que otras son inútiles y pueden ser diferentes del el objetivo principal.
Por lo tanto, el trabajo del docente es encontrar las herramientas adecuadas Meneses y Monge, (2001). Señaló:

El educador es un guía. Su dirección es dar indirectamente creando oportunidades, brindando el tiempo y el espacio necesarios, y brindando materiales y conocimiento. La forma de juego se basa principalmente en la edad del aprendiz. A la hora de elegir un juego, el educador debe considerar que la experiencia a realizar es positiva. (pp. 113-124).

Las actividades lúdicas deben tener como objetivo maximizar la diversión, la relajación y la risa espontánea. Es decir, los juegos intentan descubrir el origen de las emociones que a veces son retenidas por el formalismo rutinario, serio y poco dispuesto a socavar el plan impuesto. Meneses \& Monge, ob. cit., Menciona aportan grandes beneficios a los niños, incluidas contribuciones al desarrollo del potencial cognitivo la percepción, la activación de la memoria y el arte del lenguaje.

Los beneficios de las actividades lúdicas en el aula Sánchez (2016) apunta que por un lado, los juegos en sí tienen una motivación inherente, el deseo de obtener los mejores resultados y la competitividad que generan. Por otro lado, Córdoba, Lara y García (2017) creen que las expresiones lúdicas, especialmente el juego, están diseñadas para permitir que los niños den un significado significativo al mundo natural y social que los rodea. Con todo, la experiencia de implementar conocimientos en el aula a través de actividades lúdicas es estimulante y muy atractiva para los niños. 
Es decir, los juegos se convierten en el punto de partida para su integración, y se pueden citar algunos beneficios que ayudan a mejorar el proceso de enseñanza. Sin perder la importancia que tiene cada uno de ellos, los juegos son una herramienta indispensable Fomentar la creatividad y la imaginación, a fin de realizar el desarrollo intelectual de los niños, mejorar su autoestima y promover su desarrollo físico. Lo más importante es que los juegos son divertidos para los niños, lo que sin duda permitirá la integración de los valores sociales necesarios para el desarrollo social.

Las actividades lúdicas deben tener como objetivo cultivar la creatividad de los niños, permitiéndoles resolver los problemas cotidianos, tener su propia iniciativa $y$ desarrollar el pensamiento lógico. Es importante considerar todos los aspectos de una actividad de lúdica para poder lograr el objetivo deseado, por lo que es importante considerar lo que dijo Craty (1974). "La movilidad incómoda no garantiza un aprendizaje intelectual óptimo". Por lo tanto, al diseñar actividades de entretenimiento, se deben considerar elementos Objetivos didácticos, comportamientos entretenidos y reglas de juego". Además, (Megías y Lozano, 2000) insiste en que los juegos son muy importantes para el desarrollo integral, en el proceso de enseñanza debe ser utilizado como estrategia metodológica y debe ser lleno de diversión, motivación y creatividad.

Las actividades lúdicas deben diseñarse de acuerdo con su finalidad prevista, y adecuarse a las metas que se persiguen en el aula para lograr estas metas. Las actividades lúdicas deben encargarse de la enseñanza: metas, las reglas del juego deben ser especificadas por el instructor, la interacción entre participantes, según participación Adaptarse a la edad o personalidad de la persona para solucionar el problema es importante considerar que además de promover el aprendizaje, las actividades de entretenimiento también deben brindar: entretenimiento durante la actividad, emociones, motivación para participar en el juego, autoestima, creatividad e interacción social.

\section{Relevancia de la lectura en el aprendizaje}

El medio eficaz de adquirir conocimientos es la lectura. La lectura puede fortalecer el pensamiento lógico, desarrollar la creatividad y promover la capacidad de los niños para expresarse. Ramírez, et al. (2011) consideran que la lectura es un proceso casi indescriptible en la educación escolar. "La lectura no solo es importante en el aprendizaje, sino que también puede estimular la creatividad, relajarse $\mathrm{y}$ permitir que las personas comprendan realidades distintas y lejanas". Por tanto, el proceso de lectura no es solo un proceso obligatorio de aprendizaje, sino también una forma de ser incluidos en el mundo imaginario, en el que los libros invitan a participar.

Así insiste Prieto, (1982) manifestando que "La lectura es también una forma de vida, cuando las ideas que ayudan a actuar se extraen de la lectura y se hacen más efectivas y valiosas a través de la rica experiencia". Se puede decir que leer significa crear pensamientos racionales, permitir soñar, respetar reglas coexistentes, aprender cosas nuevas, etc.

La lectura en el aprendizaje, especialmente en la era de los rápidos avances tecnológicos. Esto es indudablemente correcto. Si se lee cada vez menos, la generación actual puede quedar excluida de una sociedad avanzada. Porque se están convirtiendo en las 
áreas principales. Donde migran la mayoría de los miembros de la sociedad actual. El objetivo no es solo aprender a leer, sino comprender lo que ha leído.

Candela, (2018) Conceptualiza la lectura comprimida como la habilidad humana que no solo incluye la capacidad de leer el texto, sino que también tiene la capacidad de comprender el contenido de la lectura y la información que el autor del libro, texto y revista quiere transmitir. Sea crítico y sepa extraer ideas principales e ideas secundarias, compararlas y sacar conclusiones. En otras palabras, aprenda a interactuar con la lectura. De hecho, la lectura integral aumenta la probabilidad de éxito de la asignatura en tareas académicas, laborales y profesionales. Por ello, Sacristán (2007) insiste en que "la lectura es una actividad compleja. Involucra muchos procesos, algunos de los cuales ocurren en la etapa inicial de procesamiento, para luego interactuar con otros procesos más complejos para extraer el significado del texto. Es decir, comprensión". Por tanto, es importante considerar lo que dijo Sacristán (2007) la lectura es una actividad compleja

Implica muchos procesos, algunos de los cuales ocurren en la etapa inicial de procesamiento, y luego interactúan con otros procesos más complejos para extraer el significado del texto. Eso es entender. Por lo tanto, es importante considerar lo que dijo Sacristán (2007): Cuando el lector se enfrenta a un texto, debe tener un sistema de conocimiento adecuado, de lo contrario no podrá entender el mensaje que el autor quiere transmitir, de lo contrario, malinterpretará lo que se está expresando.

Ya sea en la escuela o en casa, se deben desarrollar estrategias para que los niños que leen también comprendan. Este es un trabajo común que en el futuro puede evitar, por ejemplo, que alguien firme un contrato sin conocer sus verdaderas intenciones, o que el alumno se convierta en una entidad duplicada sin cuestionar lo aprendido.

Según la investigación de (Patiño, Alvarado, Zambrano y Barberan, (2017) Patiño et al., (2017) en los países de América Latina, una gran cantidad de personas no saben leer, e incluso muchas personas que han completado sus calificaciones académicas pueden leer, pero existen dificultades para comprender y comprender lo que están leyendo. Por otro lado, en el contexto ecuatoriano, Paredes, Paredes y Fonseca (2018) determinaron que la mayoría de los estudiantes que ingresan al primer semestre de la Facultad de Ciencias Psicológicas de la Universidad Central del Ecuador tienen problemas de comprensión lectora, lo que limita la complejidad de la lectura. Comprensión El desarrollo de tareas y actividades que involucran esta habilidad.

En Ecuador, según datos proporcionados por el Instituto Nacional de Evaluación Educativa (2018), una gran proporción de estudiantes que tomaron el examen PISA reflejó niveles insuficientes de lectura en el campo. Esto es preocupante porque hay evidencia de que una gran cantidad de estudiantes no alcanza el nivel básico la comprensión lectora, que afecta la calidad de su aprendizaje y la posibilidad de obtener las mejores calificaciones escolares.

El Ministerio de Educación de Ecuador ha establecido habilidades de comprensión lectora, y se deben utilizar cursos de lengua y literatura para mejorar la capacidad lectora de los estudiantes de quinto grado de educación básica.

Las limitaciones y dificultades de los estudiantes para desarrollar la lectura integral 
se debe a varios factores, uno de los principales es la estrategia metodológica adoptada por el docente. Según las instituciones educativas ecuatorianas, los docentes que utilizan métodos metodológicos tradicionales todavía tienen en general una alta tasa de prevalencia, según el estudio, los estudiantes son receptores pasivos de conocimientos que los docentes deben difundir, en lugar de un diálogo activo de construcción de conocimientos. Estudiantes, sus antecedentes y objetivos educativos.

La Escuela de Educación Primaria Numa Pompilio Llona está ubicada entre las calles San Bartolomé y San Agustín en la Parroquia de San Buenaventura, Latacún, Estado Cotopaxi. Esta institución educativa es una escuela regular y brinda apoyo económico. Servicios educativos a nivel elemental y educación básica general en el régimen de Sierra Leona a través de clases presenciales y tempranas. Cuenta con 12 docentes y 210 alumnos Por una emergencia sanitaria, el Ministerio de Educación implementa actualmente un método de aprendizaje conjunto en el hogar, y actualmente recibe servicios educativos en todos los niveles $\mathrm{y}$ subniveles.

Se puede demostrar claramente que en el proceso de enseñanza y aprendizaje de niños y niñas, las instituciones estudiadas no adoptaron estrategias ya que existe poco conocimiento sobre las actividades, la tecnología y la metodología de la innovación, es necesario realizar un trabajo adecuado según el nivel para mejorar las dificultades que se presenten. En este sentido, es necesario desarrollar un plan de actividades lúdicas para mejorar el nivel de aprendizaje. La mejora de la lectura integral, especialmente en todas las áreas del aprendizaje de los estudiantes, es propicia para el desarrollo de la inteligencia, porque en todos los niveles de aprendizaje, la tarea de un educador es permitir que los estudiantes desarrollen todas sus capacidades y alcancen su máximo potencial, de esa manera evitando que el problema futuro aparezca.

El problema descrito se puede expresar a través de las siguientes preguntas: ¿Cómo afectan las actividades lúdicas el nivel de lectura integral de los alumnos de quinto grado de la Escuela Primaria Numa Pompilio Llona? Después de evaluar de quién es el problema durante la investigación, se puede proponer otra solución basada en la situación real y las necesidades del entorno educativo y social.

Con el fin de orientar eficazmente todos los comportamientos y actividades descritos en el proceso de investigación, el propósito de este artículo es desarrollar un programa de actividades interesante para mejorar la capacidad de lectura integral de los estudiantes.

\section{MÉTODO}

Para el desarrollo y análisis de la información en el estudio se utilizó el enfoque cuali-cuantitativo, de tipo descriptivo transversal, en la de la Escuela Numa Pompilio Llona en Ecuador. El universo estuvo constituido 50 personas entre estudiantes y docentes quedando la muestra con un grupo de treinta (30) estudiantes y con la participación de doces (12) profesores. La técnica empleada fue la encuesta con el fin de recoger de manera general los criterios y las opiniones acerca de la utilidad de las actividades Lúdicas para el mejoramiento de la Lectura Comprensiva de los estudiantes de Quinto año de Educación Básica de la Escuela "Numa Pompilio Llona. 
Para el desarrollo de programas de actividades lúdicas para el mejoramiento de la lectura comprensiva en los niños de Quinto Año de Educación Básica de la Escuela Numa Pompilio Llona fueron tomada en consideración cinco (5) actividades las cuales se mencionan a continuación: Actividad 1). El cubo de mi lectura, 2). La historia desordenada. 3). La Ruleta de las fábulas, 4). La serpiente de la lectura, 5). Mi lectura a partir de imágenes y por último la actividad 6). El párame la mano de la lectura.

Se contó con la participación de tres expertos y tres usuarios para la validación del programa con una calificación de 5 que equivale a excelente. Lo que determinó la factibilidad y viabilidad para contribuir en la resolución de la problemática en torno al deficiente nivel de lectura comprensiva del educando.

\section{RESULTADOS}

Encuesta aplicada a los docentes de la Escuela de Educación General Básica "Numa Pompilio Llona"

Como se observa en la Tabla 1 del $100 \%$ de encuestados (12) se obtuvo que el $25 \%$ indicaron que el nivel de lectura es excelente, el $33 \%$ consideraron que es bueno, y finalmente el $42 \%$ manifestaron que es regular.

Tabla 1. Nivel de lectura comprensiva en general de sus estudiantes.

\begin{tabular}{lcc}
\hline Alternativas & Frecuencia & Porcentaje \\
\hline EXCELENTE & 3 & $25 \%$ \\
BUENO & 4 & $33 \%$ \\
REGULAR & 5 & $42 \%$ \\
\hline Total & 12 & $100 \%$ \\
\hline
\end{tabular}

Para conocer si utilizan actividades lúdicas, el 25\% de los docentes respondieron que si la utilizan; el $50 \%$ que a veces; mientras que el $25 \%$ manifestó que nunca realizan actividades lúdicas en sus procesos de enseñanza.

Como se muestra en la Tabla 2 de un total de 12 docentes encuestados; que representan el 100\%; manifestaron que es vital importancia la lectura.

Tabla 2. Importancia de los procesos de aprendizajes.

\begin{tabular}{lcc}
\hline Alternativas & Frecuencia & Porcentaje \\
\hline SI & 12 & $100 \%$ \\
NO & 0 & $0 \%$ \\
\hline Total & 12 & $100 \%$ \\
\hline
\end{tabular}

En la Tabla 3 se observa que el 33\% contestó que siempre recomendaran a sus compañeros que utilicen actividades lúdicas para desarrollar la lectura comprensiva, mientras que el 67\% manifestaron que a veces. 
Tabla 3. Recomendación sobre el uso de actividades lúdicas para el desarrollo de la lectura.

\begin{tabular}{lcc}
\hline Alternativas & Frecuencia & Porcentaje \\
\hline SIEMPRE & 4 & $33 \%$ \\
A VECES & 8 & $67 \%$ \\
NUNCA & 0 & $0 \%$ \\
\hline Total & 12 & $100 \%$ \\
\hline
\end{tabular}

Al preguntar acerca de la influencia del lenguaje en el desarrollo personal y social de los estudiantes, 58\% respondieron que, si influye la lectura en el lenguaje, desarrollo personal y social, mientras que el 25\% manifiesto es poca la influencia de la lectura en el desarrollo de los estudiantes, y el 17\% declara que no influye en nada. (Tabla no 4).

Tabla 4. Uso del lenguaje, el desarrollo personal y social de los estudiantes.

\begin{tabular}{lcc}
\hline Alternativas & Frecuencia & Porcentaje \\
\hline MUCHO & 7 & $58 \%$ \\
POCO & 3 & $25 \%$ \\
NADA & 2 & $17 \%$ \\
\hline Total & 12 & $100 \%$ \\
\hline
\end{tabular}

Al aplicar la encuesta a los docentes, en la pregunta sobre la consideración que tenían acerca de la importancia del desarrollo de la lectura comprensiva en sus estudiantes el 100\% refirió que es de vital importancia desarrollar la lectura comprensiva. (Tabla 5).

Tabla 5. Desarrollar la lectura comprensiva en sus estudiantes.

\begin{tabular}{lcc}
\hline Alternativas & Frecuencia & Porcentaje \\
\hline SI & 12 & $100 \%$ \\
NO & 0 & $0 \%$ \\
\hline Total & 12 & $100 \%$ \\
\hline
\end{tabular}

Al indagar sobre si le gustaría conocer las actividades lúdicas para la lectura comprensiva el cien por ciento de los docentes respondieron afirmativamente.

\section{Resultados de la encuesta a los estudiantes}

Del total de estudiantes, el 23\% responden que les gusta leer, mientras que 50\% manifiesta que les gusta leer poco, y finalmente el $27 \%$ no les gusta leer nada. (Tabla 6). 
Tabla 6. El gusto por la lectura.

\begin{tabular}{lcc}
\hline Alternativas & Frecuencia & Porcentaje \\
\hline MUCHO & 7 & $23 \%$ \\
POCO & 15 & $50 \%$ \\
NADA & 8 & $27 \%$ \\
\hline Total & 30 & $100 \%$ \\
\hline
\end{tabular}

Como se muestra en la Tabla 7 de un total de 30 encuestados que corresponde al 100\%, el $7 \%$ respondieron que les gusta leer poesía, el 70\% manifestaron que les gusta leer cuentos, el $23 \%$ adivinanzas, y el $0 \%$ ninguno.

Tabla 7. Preferencia de texto para lectura

\begin{tabular}{lcc}
\hline Alternativas & Frecuencia & Porcentaje \\
\hline POESIA & 2 & $7 \%$ \\
CUENTOS & 21 & $70 \%$ \\
ADIVINANZAS & 7 & $23 \%$ \\
NINGUNO & 0 & $0 \%$ \\
\hline Total & 30 & $100 \%$ \\
\hline
\end{tabular}

Sobre si le gustaría que su docente utilice actividades lúdicas para fomentar la lectura comprensiva, $97 \%$ responden que les gustaría que sus docentes utilicen actividades lúdicas, el $3 \%$ manifiesta que a veces y el $0 \%$ ninguno (Tabla 8 ).

Tabla 8. Utilizar actividades lúdicas para fomentar la lectura

\begin{tabular}{lcc}
\hline Alternativas & Frecuencia & Porcentaje \\
\hline SIEMPRE & 29 & $97 \%$ \\
A VECES & 1 & $3 \%$ \\
NUNCA & 0 & $0 \%$ \\
\hline Total & 30 & $100 \%$ \\
\hline
\end{tabular}

Se muestra en la Tabla 9 si comprende lo que lee, 23\% manifiestan que siempre comprenden lo que leen, mientras que el $37 \%$ responden que solo a veces comprenden lo que leen y $40 \%$ nunca entienden lo que lee.

Tabla 9. Comprende lo que lee

\begin{tabular}{lcc}
\hline Alternativas & Frecuencia & Porcentaje \\
\hline SIEMPRE & 7 & $23 \%$ \\
A VECES & 11 & $37 \%$ \\
NUNCA & 12 & $40 \%$ \\
\hline Total & 30 & $100 \%$ \\
\hline
\end{tabular}


Al relacionar con la pregunta anterior al preguntarle que, si en su casa leen revistas, periódicos, libros $\mathrm{u}$ otros textos, $13 \%$ manifiestan que, si leen en casa, mientras que el $87 \%$ responden que no leen.

En cuanto al tiempo libre que le gustaría hacer, $17 \%$ manifiestan que les gusta ayudar en casa, mientras que el $7 \%$ responden que les gusta leer y el $77 \%$ ver la televisión.

Como se observa en la tabla no $3,27 \%$ manifiestan que sus docentes si dedican tiempo para la lectura, mientras que el $60 \%$ responden que solo a veces realizan esta actividad, y el 13\% nunca. (Tabla 10).

Tabla 10. En su institución los docentes dedican tiempo para fomentar la lectura

\begin{tabular}{lcc}
\hline Alternativas & Frecuencia & Porcentaje \\
\hline SIEMPRE & 8 & $27 \%$ \\
A VECES & 18 & $60 \%$ \\
NUNCA & 4 & $13 \%$ \\
\hline Total & 30 & $100 \%$ \\
\hline
\end{tabular}

Respecto a que si lee los libros que le recomiendan sus docentes el $27 \%$ manifiestan que sus docentes si dedican tiempo para la lectura, mientras que el $60 \%$ responden que solo a veces realizan esta actividad, y el 13\% nunca.

$\mathrm{Al}$ analizar los resultados del post test se observa que el $96.6 \%$ le gusta leer siempre, el $93 \%$ comprende lo que lee, con relación a que hace en su tiempo libre la mayoría (77\%) prefieren leer un libro, $77 \%$ expresan que los docentes dedican tiempo para fomentar la lectura, y por último la totalidad de los estudiantes expresan Leer los libros que le recomiendan sus docentes. (Tabla 11).

Los resultados obtenidos de la aplicación del post test permiten apreciar que la propuesta ejecutada ha generado cambios significativos en las habilidades de comprensión lectora, logrando que el 93\% de estudiantes comprenda lo que ha leído.

Tabla 11. Resultados del post test.

\begin{tabular}{llll}
\hline Pregunta & Opción & Frecuencia & Porcentaje \\
\hline ¿Le gusta leer? & Siempre & 29 & $96,67 \%$ \\
& A veces & 1 & $3,33 \%$ \\
& Nunca & 0 & $0 \%$ \\
¿Comprende lo que lee? & Total & 30 & $100 \%$ \\
& Siempre & 28 & $93 \%$ \\
& A veces & 2 & $7 \%$ \\
& Nunca & 0 & $0 \%$ \\
& Total & 30 & $100 \%$ \\
\hline
\end{tabular}




\begin{tabular}{llll}
\hline Pregunta & Opción & Frecuencia & Porcentaje \\
\hline ¿Qué le gusta realizar en su tiempo libre? & Ayudar en casa & 2 & $7 \%$ \\
& Leer libros & 23 & $77 \%$ \\
& Ver televisión & 5 & $16 \%$ \\
& Total & 30 & $100 \%$ \\
& & & \\
¿En su institución los docentes dedican & Siempre & 22 & $73 \%$ \\
tiempo para fomentar la lectura? & A veces & 8 & $27 \%$ \\
& Nunca & 0 & $0 \%$ \\
¿Lee los libros que le recomiendan & Total & 30 & $100 \%$ \\
docentes? & Siempre & 30 & $100 \%$ \\
& A veces & 0 & $0 \%$ \\
& Nunca & 0 & $0 \%$ \\
& Total & 30 & $100 \%$ \\
\hline
\end{tabular}

\section{DISCUSIÓN}

La lectura es un factor fundamental para el aprendizaje en todas las personas, dado que a través de ella es posible reconocer diferentes temáticas, teorías y presunciones que permiten el acercamiento a el conocimiento, dando también la posibilidad de conocer el mundo a partir de las letras.

Urgilés, (2019) planteó en su tesis que uno de los grandes desafíos que enfrentan en la actualidad los docentes, es desarrollar y/o fortalecer en los estudiantes de enseñanza media la adquisición de habilidades comunicativas que les permitan ser hablantes y lectores competentes. Para ello, se realizan innumerables esfuerzos, tanto en la búsqueda de una metodología de trabajo innovadora y eficaz, como en la selección de textos que respondan a las expectativas de los estudiantes, con el propósito de orientarlos en su aprendizaje de la Lectura y la escritura. Para fines de este estudio se pudo comprobar que la lúdica contribuye de manera eficaz al mejoramiento de la comprensión de textos de los estudiantes de Quinto año de Educación Básica de la Escuela "Numa Pompilio Llona", proporcionando elementos conceptúales que ayuden a concretar, aterrizar y ampliar las actitudes académicas ofreciéndoles nuevas posibilidades de desarrollo del ser a partir de su propio interés.

Seguidamente se comparan la investigación Ardila, Castaño, \& Tamayo, ( 2015) el cual refiere que los estudiantes manifestaron en varias ocasiones el deseo de explorar la comprensión lectora desde ámbitos lúdicos como el juego, el teatro, la música, entre otros. Se hizo notar el deseo por parte de los educandos, de nuevas estrategias que los acerquen de manera positiva y divertida al acto lector y comprensivo.

En una línea similar Benavides \& Tovar, (2017), en su investigación demostró que la puesta en práctica de las estrategias de comprensión lectora permitió establecer que algunos docentes de grado tercero de la ENSUP poseían debilidades en la fundamentación de sus procesos teóricos $y$ en otros se 
presentaban deficiencias en la estructuración de un plan pertinente de comprensión lectora lo anterior afectaba la orientación que se brindaba a los estudiantes respecto de la forma de los procedimientos apropiados para alcanzar no solo el desarrollo de la comprensión sino el de la competencia comunicativa. En este estudio se pudo evidenciar que los docentes están conscientes sobre la importancia de desarrollar la lectura comprensiva en sus estudiantes en todos los niveles y así generar aprendizajes significativos.

Cunachi \& Leyva, (2018) en su tesis abordan la comprensión lectora y su incidencia en el aprendizaje en el aspecto de la comunicación integral, con los estudiantes del nivel de educación básica donde se determinó la existencia de una relación directa y significativamente alta entre la comprensión lectora y el nivel de aprendizaje de los estudiantes. Estudio que afianzó los resultados manifestado en este manuscrito, basado en las estrategias para lograr la comprensión lectora en estudiantes.

En este mismo orden y dirección, Molina (2016) en su investigación acerca de las destrezas de lectura comprensiva en los estudiantes de bachillerato, asume la comprensión lectora como la capacidad de entender el significado que adquieren los elementos que conforman el texto escrito, lo cual constituye una habilidad relevante dentro del proceso educativo. En donde se arrojó como resultado que la mayoría de los docentes no desarrollan destrezas de la lectura comprensiva ni aplican estrategias cognitivas que contribuyen a comprender analizar y producir textos. Coincidiendo con este estudio.

Igualmente, Cruz (2018) en su investigación acerca de la lúdica como estrategia didáctica para el mejoramiento de la comprensión lectora, se propone como objetivo contribuir en el fortalecimiento de la comprensión lectora a través de la implementación de las estrategias lúdicas con los estudiantes. En donde se evidenció que el aporte positivo de las estrategias lúdicas, afianzando el grupo de actividades que se propusieron en este estudio para la mejora de la comprensión lectora.

De la misma manera, Reyes, (2016) indaga sobre la aplicación de las actividades lúdicas en el aprendizaje de la lectura enfocado a los niños de Educación Primaria con el objetivo de diseñar y aplicar un plan estratégico que permita promover el uso de los juegos didácticos como recurso metodológico para la enseñanza de la lectura para ello se aplica una metodología de investigación cuantitativa cualitativa por medio de la aplicación del método científico a través de las etapas conceptual de diseño y planeación empírica analítica y de difusión de resultados. Con esta investigación se coinciden con relación a los niveles educativos y la necesidad de aplicación de didácticas pedagógicas para la mejora en la comprensión lectora.

En este orden de ideas se puede citar Chicaiza, (2015) en su tesis demostró el déficit en cuanto a las estrategias lúdicas para fomentar la comprensión lectora. Por su parte, Linero \& Macea, (2018) en su investigación aborda en la lectura lúdica libre como herramienta didáctica para fortalecer la comprensión lectora enfocándose en los estudiantes, en donde determinó que los estudiantes presentan deficiencias en el nivel de comprensión literal inferencial y crítico, lo cual afecta su proceso de aprendizaje, realidad semejante a la con la población que se trabajó en este estudio.

Linero \& Macea, (2018). La lectura lúdica libre como herramienta didáctica para 
fortalecer la comprensión lectora. Tesis de Maestría. Montería: Universidad Pontificia Bolivariana.

Las investigaciones analizadas, permiten evidenciar la importancia de la lectura comprensiva, lo cual sustenta la pertinencia y relevancia de la presente investigación además reflejan y corroboran la problemática del deficiente desarrollo de la lectura comprensiva en los estudiantes, interrelacionada con causas pedagógicas como la falta de conocimientos, la aplicación de estrategias metodológicas tradicionales, monótonas, la falta de innovación, creatividad y motivación en las actividades didácticas empleadas por los docentes dentro del proceso de enseñanzaaprendizaje. Comparando los alcances del estudio presentado con los de este se hace urgente la aplicación de nuevas didácticas innovadoras, tecnológicas para mejorar el proceso de enseñanza y aprendizaje dentro de la comprensión lectora.

Basado en los datos que fueron obtenidos y analizados por investigaciones antes mencionadas se plantea que la lectura comprensiva es la base primordial en cada proceso de enseñanza y aprendizaje que los docentes imparten en su labor pedagógica, siempre que esta se aplique usando métodos verificados $y$ que estos hayan recogidos grandes alcances en el quehacer pedagógico, específicamente relacionados con el tema de lectura comprensiva.

\section{CONCLUSIONES}

Para finalizar a lo largo del desarrollo del estudio y basado en los resultados obtenidos se llegó a las siguientes conclusiones:

La utilización de estrategias lúdicas a los estudiantes debe ser una opción a elegir en en la población estudiada, debido a que se identificó la carencia y falta de motivación para desarrollar habilidades dentro de la comprensión lectura.

Es importante el desarrollo y elaboración de un Programa de Actividades Lúdicas para el mejoramiento de la lectura comprensiva basado en fundamentos esenciales en las diferencias psicosociales de los estudiantes a los cuales esté dirigido el programa de enseñanza. Por lo que se sugieren poner en práctica las actividades sugeridas en este estudio.

\section{REFERENCIAS}

Ardila, L. M., Castaño, A., \& Tamayo, G. E. (2015). Estartegias lúdico pedagógicas para el fortaleciendo de la comprensión lectora. . Armenia, Quindío : Fundacion Universitaria los Libertadores

Benavides, C. F., \& Tovar, N. (2017). Estrategias Didácticas para Fortalecer la Enseñanza de la Comprensión Lectora en los Estudiantes del Grado Tercero de la Escuela Normal Superior de Pasto . San Juan: Universidad Santo Tomás

Candela, F. ((2018). La lectura comprensiva: un instrumento para aprender ciencias. Revista Praxis, Educación y Pedagogía

Chicaiza, M. (2015). Diseño de una estrategia lúdica para mejorar la comprensión lectora en Educación General Básica. Tesis de Maestría. Ambato, Ecuador: Pontificia Universidad Católica del Ecuador

Córdoba, E., Lara, F., \& García, A. (2017). El juego como estrategia lúdica para la educación inclusiva del buen vivir. ENSAYOS. Revista de la Facultad de Educación de Albacete, 32(1), 81-92

Craty, B. (1974). Juegos didácticos activos. . México D.F: Pax México

Cruz, O. (2018). La lúdica, una estrategia para la comprensión lectora. Tesis de Maestría. Bogotá, Colombia: Universidad Externado de Colombia 
Cunachi, G., \& Leyva, G. (2018). Comprensión lectora y el aprendizaje en el área de Comunicación Integral en los estudiantes de Educación Básica Alternativa de las instituciones educativas del distrito de Chaclacayo UGEL 06 Ate-Vitarte año 2015. Tesis de Grado. La Cantuta: Universidad Nacional de Educación Enrique Guzmán y Valle

Domínguez, C. (2015). La lúdica: Una estrategia pedagógica depreciada. . Ciudad Juárez, Chiguagua: Universidad Autónoma de Ciudad Juarez

Huizinga, J. (1968). Homo ludens. Buenos Aires: Emecé Editores S.A.

Instituto Nacional de Evaluación Educativa. (2018). Resultados de PISA para el desarrollo. Quito, Ecuador: Ediciones públicas

Linero, L., \& Macea, A. (2018). La lectura lúdica libre como herramienta didáctica para fortalecer la comprensión lectora. Tesis de Maestría. Montería: Universidad Pontificia Bolivariana.

Megías, A., \& Lozano, L. (2000). El juego infantil y su metodología. Madrid: l Editex S.A.

Meneses, M., \& Monge, M. (2001). El juego en los niños: enfoque teórico. Educación, 25(2), 113-124

Molina, L. (2016). Destrezas en la lectura comprensiva en los estudiantes de primero de bachillerato. Tesis de Maestría. Esmeraldas, Ecuador: Pontificia Universidad Católica del Ecuador

Paredes, M. T., Paredes, E., \& Fonseca, S. (2018). Niveles de lectura comprensiva de los estudiantes de los primeros semestres de la Facultad de Ciencias Psicológicas de la
Universidad Central Del Ecuador2(2),. Retos de la ciencia, 2(2), 75-90

Patiño, V., Alvarado, A., Zambrano, M., \& Barberan, J. (2017). La lectura comprensiva en el desarrollo intelectual de los niños y niñas en la educación general básica media. Polo de conocimiento, 2(7), 215-225

Prieto, L. (1982). La magia de los libros. Caracas,: Monte Ávila Editores

Ramírez, N., Díaz, M., Reyes, P., \& Cueca, O. (2011). Educación lúdica: una opción dentro de la educación ambiental en salud. Seguimiento de una experiencia rural colombiana sobre las geohelmintiasis. Revista Med, 23-36

Reyes, T. (2016). Aplicación de las actividades lúdicas en el aprendizaje de la lectura en niños de educación primaria. Tesis Doctoral. Isla de Margarita, Venezuela: Universidad de Córdoba

Sacristán, F. (2007). La lectura como instrumento clave en el aprendizaje escolar.(Brasil). Práxis Educativa, 2(1), 1326

Sánchez, S. (2016)). La importancia del juego en el proceso de enseñanzaaprendizaje de una lengua extranjera. España: Universidad de Valladolid

Urgilés, K. D. (2019). Estrategias lúdicas para desarrollar la lectoescritura. Guayaquil: Universidad Católica de Santiago de Guayaquil

Zuluaga, C., \& Gómez, M. (2016). Metodología lúdica para la enseñanza de la programación dinámica determinista en un contexto universitario. Entramado, 12(1), 236-249 